\title{
THE DETERMINATION OF ABELIAN HALL SUBGROUPS BY A CONJUGACY CLASS STRUCTURE
}

\author{
WOLFGANG KimMERLE AND ROBERT SANDLiNG
}

Pere Menal in memoriam

\begin{abstract}
The object of the article is to show that a Jordan-Hölder class structure of a finite group determines abelian Hall subgroups of the group up to isomorphism. The proof uses the classification of the finite simple groups.
\end{abstract}

A conjugacy class structure on a group captures information about its normal subsets, for example, that which is deducible from its character table or from its integral group ring. Various such structures were introduced in [KS] and used there to draw conclusions about the group previously invcstigated only using the character table or the integral group ring.

The most basic class structure considered was a Jordan-Hölder class structure, one which captures the poset of normal subscts of a group, records their sizes, indicates which normal subsets arc normal subgroups and which are the preimages of the conjugacy classes of its quotient groups.

It was shown in $[\mathbf{K S}]$ that such a class structure determines the chief factors of a group. More technically, groups $G$ and $G *$ have the same chief factors if they are in class correspondence of type $J H$, that is, if there is a bijective correspondence between them preserving normal subsets and their assumed properties. (More formal definitions are available in our earlier paper.)

It was also shown that the isomorphism type of an abelian Sylow subgroup is determined by a class structure of type $J H$, and that of an abelian Hall subgroup by a stronger class structure (namely, one in which the set of primes involved in the orders of the elements in conjugacy classes is also posited). In this paper, this extra hypothesis is removed to give the following. 
Main Theorem. A Jordan-Hölder class structure determines abelion Holl subgroups up to isomorphism.

It should be noted that the results of our earlier paper are dependent on the classification of the finite simple groups. Apart from building on our earlier results, this paper makes further invocations of the classification.

Our collaboration on the topic of abelian Sylow and Hall subgroups was a direct consequence of the second author's participation [San] in the 1986 ring theory conference in Granada in which Pere Menal played a prominent role.

We begin the proof of our theorem by fixing some notation. The groups $G$ and $G$ * will be assumed to be in class correspondence of type $J H$ with $*$ being used to denote the bijection (on subsets and subgroups as well as on elements). We assume that, for a fixed set $\pi$ of primes, $G$ has abelian Hall $\pi$-subgroups. We must show that $G *$ also has abelian Hall $\pi$-subgroups and that they are isomorphic to those of $G$. By our result on abelian Sylow subgroups, it suffices to show that $G *$ has Hall $\pi$-subgroups and that they are abelian (or merely nilpotent).

The proof of the determination of abelian Hall $\pi$-subgroups when $|\pi| \geq 2$ is more elaborate than that for abelian Sylow subgroups. One reason for this is the absence of a direct analogue for groups with abelian Hall $\pi$-subgroups of the criterion [KS, 2.1] for detecting abclian Sylow subgroups. This can be seen from the group obtained by extending $L_{2}\left(7^{5}\right)$ by the cyclic group $C_{5}$ acting as field automorphisms (here $\pi=\{3,5\}$ ). (Contrast the behaviour of Sylow subgroups as seen in the Proposition below with that of Hall subgroups.)

Under $a$ class correspondence of type $J H$, the orders of $x$ and $x *$ need not coincide (viz, the quaternion and dihedral groups of order 8). This renders subgroups like $O^{\pi^{\prime}}(G)$, generated by all $\pi$-elements of $G$, less useful than they would be if orders were preserved as they are by stronger class correspondences (see [KS]). In their place, we use the following characteristic subgroup of a group.

Definition. Let $Z$ be a group and $\rho$ a set of primes. Define $C_{\rho}(Z)$ as the subgroup generated by all $x$ in $Z$ for which $\left|Z: C_{Z}(x)\right|$ is a $\rho^{\prime}$-number, where $C_{Z}(x)$ denotes the centralizer of $x$ in $Z$.

Under a class correspondence of type $J H, C_{\pi}(G *)=C_{\pi}(G) *$. Note, in addition, that, if $G$ has abelian Hall $\pi$-subgroups, then they are all contained in $C_{\pi}(G)$; it follows from a theorem of Wiclandt [Suz, 5.3.2] that $O^{\pi^{\prime}}(G) \leq C_{\pi}(G)$.

In the proof of Theorem 2.1 of [KS], elements normalising each simple 
factor of each perfect minimal normal subgroup of a group were examined. For the determination of abelian Hall subgroups, such elements play a more prominent role which is made more explicit in the following definition of the characteristic subgroup $K(G)$ which they comprise. If the group $G$ has a unique minimal normal subgroup and this subgroup is nonabelian (so that $G$ is embedded in a wreath product [Rose, p.223]), $K(G)$ is the intersection of $G$ with the base group of the wreath product.

Definition. The characteristic subgroup $K(G)$ of $G$ is defined as the intersection of all $N_{G}(S)$ where $S$ is a nonabelian simple subgroup of $G$ which is normal in Soc $G$, the socle of $G$.

For a nonabelian minimal normal subgroup $M$ of $G$, let $K\left(G \bmod C_{G}(M)\right)$ denote the inverse image in $G$ of $K\left(G / C_{G}(M)\right)$. It is easy to see that $K\left(G \bmod C_{G}(M)\right)$ is the intersection of all $N_{G}(S), S$ a simple subgroup of $G$ normal in $M$. If follows that $K(G)$ is the intersection of all such $K\left(G \bmod C_{G}(M)\right)$. The definition of $K(G)$ is similar to that of McBride's $\hat{K}(G)[\mathrm{McB}$, p.217], and the two subgroups coincide in the case, important here in reduction arguments, where $G$ has a unique and nonabelian minimal normal subgroup.

One well-known consequence of the classification of the finite simplc groups which was used in $[\mathbf{K S}]$ is needed again here, and is stated for the reader's convenience (cf. [GL, 7.10]).

Proposition. Let $S$ be a simple group of order divisible by a prime $p$. If $X$ is a subgroup of Aut $S$ in which $S \leq X$ and $p$ divides the index of $S$ in $X$, then $X$ has nonabelian Sylow $p$-subgroups.

The determination of abelian Hall subgroups is accomplished through a series of reduction steps (cf. the proof of the main theorem). They lead to the following situation which is exploited in the subsequent lemmas.

Lemma 1. Suppose that

(i) $G$ has abelian Hall $\pi$-subgroups;

(ii) each minimal normal subgroup $M$ of $G$ has order divisible by some prime in $\pi$;

(iii) each minimal normal subgroup $M$ of $G$ is nonabelian.

Then $C_{\pi}(G) \leq K(G)$ and $C_{\pi}(G *) \leq K(G *)$.

Proof: For the first conclusion, it suffices to show that $C_{\pi}(G) \leq$ $K\left(G \bmod C_{G}(M)\right)$ for each minimal normal subgroup $M$ of $G$. Let $x$ be an clement of $G$ for which $\left|G: C_{G}(x)\right|$ is a $\pi^{\prime}$-number. By (ii), there is some $p \in \pi$ for which $M$ contains nontrivial Sylow $p$-subgroups. Let $P$ be a Sylow p-subgroup of $G$ contained in $C_{G}(x)$. Then $P \cap M$ is a 
nontrivial Sylow p-subgroup of $M$. It follows that, if $S$ is a simple subgroup of $G$ normal in $M$, then $x$ fixes a nontrivial element of $S$. Thus, $x \in N_{G}(S)$, which suffices.

The second conclusion follows in a similar way using the result from [KS] that each minimal normal subgroup $M *$ of $G *$ is isomorphic to such a subgroup of $G$ (namely $M$ ) so that parts (ii) and (iii) apply to $M *$.

While Soc $G *=(\operatorname{Soc} G) *$ and is isomorphic to $\operatorname{Soc} G$ by $[\mathbf{K S}]$ in this case, we do not know whether similar statements hold for $K(G)$ (for example, under part (iii) above). Were such statements true, the determination of abclian Hall subgroups would be accomplished at this point. In their stead, we turn to a closer examination of two questions: when does a group $X, S \leq X \leq$ Aut $S$ for a simple group $S$, have an abelian Hall subgroup? How can this be recognised using properties detcctable under a class correspondence of type $J H$ ?

Suppose that $G$ has the unique minimal normal subgroup $M, M \approx S^{a}$, for the nonabelian simple group $S$; then $G$ may be identified with a subgroup of Aut $S^{a}$ which is a wreath product of Aut $S$ and the symmetric group of degree $a$. As remarked, $K(G)$ is the intersection of $G$ with the base group (Aut $S)^{a}$. In fact, therc is a subgroup $X$ of Aut $S$, namely the image of $N_{G}(S) / C_{G}(S)$, for which $K(G)$ is a subgroup of $X^{a}$ whose projection to each component is surjective.

The next two lemmas enable us to determine the isomorphism type of an abelian Hall subgroup of $X^{a}$ on the basis of information deducible under a class correspondence of type $J H$. It will be convenient to use two items of standard notation involving a set $\rho$ of primes and a group $Z:|Z|_{\rho}$ for the $\rho$-part of the order of $Z$ and $Z_{\rho}$ for a Hall $\rho$-subgroup of $Z$. The first of the lemmas makes another appeal to the classification of finite simple groups.

Lemma 2. Let $S$ be a finite simple group and let $Y$ be a subgroup of Aut $S$ containing $S$. Let $k$ be a divisor of $|Y|$ which is relatively prime to $|S|$. Then $Y$ has a unique conjugacy closs of subgroups of order $k$ and these subgroups ore cyclic.

Proof: For alternating and sporadic groups, whose outer automorphism groups are 2-groups, the statement is vacuous. Cyclic simple groups have cyclic outer automorphism groups so the lemma is straightforward.

For a simple group $S$ of Lie type, only a field automorphism has order relatively prime to $|S|$ (sce, for example, [McB, Lemma 4.I]). Let $\sigma$ be the set of prime divisors of $k$. Now Out $S$ has a cyclic subgroup 
(of field automorphisms) which contains a cyclic Hall $\sigma$-subgroup. As Out $S$ is soluble, $Y / S$ contains a cyclic Hall $\sigma$-subgroup, and so $Y$ does as well. By the theorem of Wielandt cited earlier, all Hall $\sigma$-subgroups of $Y$ are conjugate and every subgroup of order $k$ is contained in a Hall $\sigma$-subgroup so that these subgroups are also conjugate in $Y$.

Lemma 3. Let $S$ be a finite simple group. Suppose that $X$ and $Y$ are subgroups of Aut $S$ containing $S$ and that $|X|_{\pi}=|Y|_{\pi}$. Then $X$ has abelian Hall $\pi$-subgroups if and only if $Y$ does; if $X_{\pi}$ and $Y_{\pi}$ are abelian Hall $\pi$-subgroups of $X$ and $Y$ respectively, then $X_{\pi}$ and $Y_{\pi}$ are conjugate in Aut $S$.

Proof: Let $\sigma=\pi-\pi(S)$ and let $k=|X|_{\sigma}=|Y|_{\sigma}$. Suppose that $X_{\pi}$ is an abelian Hall $\pi$-subgroup of $X$. By the Proposition, $|X: S|=k$. By Lemma 2, $X_{\pi}=S_{\pi} C$ where $S_{\pi}=S \cap X_{\pi}$ and where $C$ is cyclic of order $k$. Lemma 2 also shows that $Y$ has a subgroup $D$ of order $k$ and that there is an automorphism $\alpha \in$ Aut $S$ such that $D=C^{\alpha}$. It follows that $X^{\alpha_{\pi}}$ is a Hall $\pi$-subgroup of $Y$. That all such Hall $\pi$-subgroups are conjugate in $Y$ follows from the previously cited theorem of Wielandt. Thus, $X_{\pi}$ and $Y_{\pi}$ are conjugate in Aut $S$.

Proof of Main Theorem: Recall that our object is to show that $G *$ has an abelian Hall $\pi$-subgroup. We may assume that $O_{\pi^{\prime}}(G)=$ $1=O_{\pi^{\prime}}(G *)$. Note that $O^{\pi^{\prime}}(G *)=O^{\pi^{\prime}}(G) *$; as $O^{\pi^{\prime}}(G) \leq C_{\pi}(G)$, $O^{\pi^{t}}(G) * \leq C_{\pi}(G) *=C_{\pi}(G *)$ so that $C_{\pi}(G *)$ contains all $\pi$-elements of $G *$.

By induction, we may assume that any proper quotient of $G *$ has abelian Hall $\pi$-subgroups. We may also assume that $G$ and $G *$ are not simple by $[\mathrm{KS}]$. Let $M$ be a minimal normal subgroup of $G$ so that $G * / M *$ has an abelian Hall $\pi$-subgroup. As $M * \approx M, M *$ also has an abelian Hall $\pi$-subgroup. By the theorem of Wielandt cited above and a theorem of Hall [Suz, 5.3.12], $G *$ has a Hall $\pi$-subgroup. By our carlier Sylow result, each Sylow $p$-subgroup for $p \in \pi$ is abelian; it remains to show that a Hall $\pi$-subgroup is itself abelian. As its image in any proper quotient of $G *$ is abelian, we may assume that $M *$ is the unique minimal normal subgroup of $G *$ and thus that $M$ is the same in $G$.

If $M$ and $M *$ are abelian, then, for some $p \in \pi$, they are (elementary abelian) p-groups. For $x * \in G *$ such that $\left|G *: C_{G *}(x *)\right|$ is $\pi^{\prime}, M * \leq$ $C_{G *}(x *)$. As these elements generate $C_{\pi}(G *), M *$ centralises $C_{\pi}(G *)$ whence $M *$ commutes with all $\pi$-elements of $G *$. Thus $M *$ is a central subgroup of each Hall $\pi$-subgroup of $G *$.

Let $H *$ be a Hall $\pi$-subgroup of $G *$. By induction, $H * / M *$ has an abelian Hall $\sigma$-subgroup $L * / M *$ for $\sigma=\pi-\{p\}$. As $M *$ is of coprime 
index in $L *$, it has a complement $K *$ which is an abelian Hall $\sigma$-subgroup of $G *$. Let $P *$ be a Sylow p-subgroup of $G *$ contained in $H *$. As $M * \leq P *, K *$ normalises $P *$. Moreover, $K *$ stabilises the normal series $P * \geq M * \geq 1$ so that $K *$ centralises $P_{*}[\mathrm{Suz}, 4.1 .13]$. Thus $H *$ is abelian as required.

Suppose, then, that $M$ and $M *$ are nonabelian. By Lemma $1, K(G)$ contains all Hall $\pi$-subgroups of $G$. As described above, $K(G)$ is isomorphic to a subgroup of $X^{a}$ where $M \approx S^{a}, S \leq X \leq$ Aut $S, K(G)$ projects onto each direct factor $X$ and $X$ has abelian Hall $\pi$-subgroups.

In a similar manner $K\left(G_{*}^{*}\right)$ is isomorphic to a subgroup of $Y^{a}$ where $M * \approx M \approx S^{a}, S \leq Y \leq$ Aut $S$ and $K(G *)$ projects onto each direct factor $Y$. By Lemma I, $C_{\pi}(G *) \leq K(G *)$. But $C_{\pi}(G *)=C_{\pi}(G) *$ and $|G|_{\pi}=\left|C_{\pi}(G)\right|_{\pi}$ so that $K(G *)$, being normal, contains a Hall $\pi$-subgroup of $G *$. We show that it is abelian by proving that $Y$ has abelian Hall $\pi$-subgroups. For this, it suffices by Lemma 3 to show that $|X|_{\pi}=|Y|_{\pi}$.

For each $p \in \pi \cap \pi(S), X$ and $Y$ have abelian Sylow $p$-subgroups, in the latter case because $Y$ is the image of $K(G *)$ which has abelian Sylow $p$-subgroups. By the Proposition, $X_{p}$ and $Y_{p}$ are contained in $S$ so that $|X|_{p}=|S|_{p}=|Y|_{p}$.

It remains to show that $|X|_{\sigma}=|Y|_{\sigma}$ for $\sigma=\pi-\pi(S)$. By Lemma $2, X_{\sigma}$ is cyclic so that $|X|_{\sigma}=\exp X_{\sigma}$. As $K(G) \leq X^{a}$ and as $K(G)$ projects onto $X, \exp X_{\sigma}=\exp K(G)_{\sigma}$. Also, $\exp K(G)_{\sigma}=\exp G_{\sigma}=$ $\exp (G / M)_{\sigma}$ since $\sigma \cap \pi(M)$ is empty.

As in the previous case $G / M$ and $G * / M *$ have isomorphic abelian Hall $\sigma$-subgroups so that $\exp (G / M)_{\sigma}=\exp (G * / M *)_{\sigma}$. As before, $\exp (G * / M *)_{\sigma}=\exp G *_{\sigma}=\exp K(G *)_{\sigma}$. Finally, $Y$ has cyclic Hall $\sigma$-subgroups by Lemma 2 and again $\exp K(G *)_{\sigma}=\exp Y_{\sigma}=|Y|_{\sigma}$. Tracing through the equalities, we conclude that $|X|_{\sigma}=|Y|_{\sigma}$ as required.

It may be possible to recognise whether a group has nilpotent Hall subgroups under a class correspondence of type $J H$. The proof above in the $M$ abelian case, or an easy argument using the Fitting subgroup, provides the following result as evidence.

Proposition. A class correspondence of type JH determines whether or not Hall subgroups of a soluble group are nilpotent. 


\section{References}

[GL] Gorenstein, D. And Lyons, R., The local structure of finite groups of characteristic 2 type, Mem. Amer. Math. Soc. 42, no. 276 (1983).

[KS] Kimmerle, W. And SANdling, R., Group theoretic and group ring theoretic determination of certain Sylow and Hall subgroups and the resolution of a question of R. Brauer, J. Algebra (to appear).

[McB] MCBRIDE, P., Nonsolvable signalizer functors on finite groups, J. Algebra 78 (1982), $215 \cdots 238$.

[Rose] Rose, J. S., "A course on group theory," Cambridge University Press, Cambridge, 1978.

[San] SANDLING, R., "A proof of the class sum correspondence using the real group algebra," Ring theory (Granada 1986), 237-244, Lecture Notes in Math. 1328, Springer, Berlin, 1988.

[Suz] SuzUkI, M., "Group theory II," Springer, New York, 1986.

Wolfgang Kimmerle:

Math. Institut B

Universität Stuttgart

7 Stuttgart 80

GERMANY
Robert Sandling:

Mathematics Department

The University

Manchester

M13 9PI.

ENGLAND

Rebut el 9 de Gener de 1992 